08

\title{
Время инкубации гетерогенного роста островков в режиме неполной конденсации
}

\author{
() В.Г. Дубровский
}

Санкт-Петербургский академический университет Физико-технический институт им. А.Ф. Иоффе РАН, Санкт-Петербург Санкт-Петербургский национальный исследовательский университет информационных технологий, механики и оптики E-mail: dubrovskii@mail.ioffe.ru

Поступило в Редакцию 29 апреля 2016 г.

Проведен теоретический анализ времени инкубации, необходимого для начала роста поверхностных островков на гетерогенных центрах нуклеации, в зависимости от газового потока материала и температуры поверхности. Показано, что при гетерогенном росте в режиме неполной конденсации время инкубации увеличивается с температурой по аррениусовскому закону и уменьшается с потоком обратно пропорционально последнему независимо от механизма диффузионного транспорта в островки. Проведено качественное сопоставление результатов с экспериментальными данными по времени инкубации трехмерных островков $\mathrm{GaN}$, возникающих на начальном этапе самоиндуцированного роста нитевидных нанокристаллов $\mathrm{GaN}$.

Исследования начальной стадии нуклеации поверхностных островков различной природы и в особенности времени инкубации (ВИ) (задержки роста с момента начала эпитаксиального процесса) имеют долгую историю [1-3]. Теоретическое рассмотрение обычно проводится в рамках кинетических моделей необратимого роста начиная с критического размера, предполагаемого постоянным [4]. Длительная инкубация ростового процесса характерна для режима неполной конденсации тонких пленок [4-6], в котором лишь малая часть осаждаемого материала остается на поверхности, а большая часть десорбируется. Модели с фиксированным критическим размером обычно приводят к зависимости ВИ $t_{i n c}$ от потока $I$ и температуры $T$ вида $t_{i n c} \propto I^{-p} \exp \left(-E / k_{\mathrm{B}} T\right)$, т.е. убывание с увеличением потока по степенному закону и аррениусовское возрастание при увеличении температуры с некоторой 
энергией активации $E$ [1-3]. Индекс $p$ неочевидным образом связан с критическим размером (точнее, с числом атомов в критическом зародыше), и этот вопрос до сих пор дискутируется [7]. По физическому смыслу, повышение температуры приводит к экспоненциальному увеличению скорости десорбции, а повышение потока - к увеличению поверхностной концентрации адатомов и, следовательно, вероятности нуклеации, что качественно объясняет приведенную выше зависимость.

В последнее время интерес к исследованиям задержек нуклеации возрос в связи с развитием методов синтеза нитевидных нанокристаллов (ННК) $\mathrm{GaN}$ на поверхностях $\mathrm{AlN} / \mathrm{Si}(111), \mathrm{Si}_{x} \mathrm{~N}_{y} / \mathrm{Si}(111)$ и $\mathrm{Al}_{x} \mathrm{O}_{y} / \mathrm{Al}_{2} \mathrm{O}_{3}$ [7-10]. В отличие от классических ННК III-V, обычно выращиваемых по механизму „пар-жидкость-кристалл“ [11], HHK GaN выращиваются по самоиндуцированному механизму - вначале на поверхности образуются трехмерные островки $\mathrm{GaN}$, которые растут изотропно до определенного размера $(\sim 5 \mathrm{~nm})$, а затем трансформируются в анизотропные ННК [12-16]. Данный метод является актуальным с точки зрения интеграции оптических гетероструктур на основе GaN с радикально пониженной плотностью дислокаций кремниевой электронной платформой $[17,18]$. Очевидно, характеристики HHK GaN на начальном этапе во многом определяют морфологию ансамблей ННК.

In situ диагностика методом дифракции быстрых электронов на отражение или квадрупольной масс-спектрометрии при молекулярнопучковой эпитаксии позволяет прецизионно определять ВИ и измерять их зависимость от потока галлия и температуры поверхности [7-10]. Значения ВИ составляют десятки или даже сотни минут. Такие длительные задержки роста связаны с используемыми для роста HНК GaN высокими температурами $\left(750-800^{\circ} \mathrm{C}\right)$ и потоками азота [14-16]. Полученные экспериментальные зависимости в целом укладываются в теоретическую, со степенным индексом $p=1.5$ на аморфном подслое $\mathrm{Si}_{x} \mathrm{~N}_{y}$ [7,9] и в пределах от 0.7 до 2 на прочих поверхностях. Трудно предположить, что абсолютно во всех случаях критический размер, являющийся функцией многих переменных, включая $I$ и $T$ [5], лежит в этих пределах. Остаются неизвестными и другие параметры, определяющие зависимость ВИ от температуры и потока. Поэтому в настоящей работе мы исследуем ВИ в частном случае гетерогенной нуклеации, приводим явное выражение для ВИ и показываем, что ВИ (по крайней мере, для данной модели) не имеет отношения к критическому размеру. 
Рассматривается случай образования островков на гетерогенных центрах (примесях, изломах ступеней [19] и т.д.) данной поверхностной концентрации $N_{0}$. Предполагается, что процесс роста лимитирован поверхностной кинетикой элемента группы III, что наверняка справедливо для островков $\mathrm{GaN}$, получаемых в обогащенных по азоту условиях [7-10,12-16]. Кинетические уравнения баланса для концентрации свободных центров нуклеации $n_{0}$ и адатомов галлия $n_{A}$ в случае необратимого роста имеют вид [20]

$$
\begin{gathered}
\frac{d n_{0}}{d t}=-D n_{A} \sigma_{0} n_{0}, \\
\frac{d n_{A}}{d t}=I-\frac{n_{A}}{t_{A}}-D n_{A} \sum_{s=0}^{\infty} \sigma_{s} n_{s} .
\end{gathered}
$$

Здесь $D$ есть коэффициент диффузии адатома, $I$ - поток галлия, $t_{A}-$ характерное время десорбции галлия и $\sigma_{s}-$ коэффициенты захвата, зависящие от числа атомов галлия („размера“) в островке. Все физические параметры в уравнениях (1) и (2) зависят от типа поверхности. Первое уравнение означает, что концентрация свободных центров уменьшается со временем в процессе присоединения к ним адатомов. Второе уравнение описывает изменение во времени концентрации адатомов за счет их адсорбции-десорбции и присоединения к островкам любого размера, включая свободные центры нуклеации при $s=0$. В случае гетерогенного роста полная концентрация всех островков равна полной концентрации центров нуклеации за вычетом концентрации свободных центров. Поэтому скорость нуклеации островков есть $d N / d t=-d n_{0} / d t$. Отметим, что суммирование в (2) может начинаться не с нуля, а с некоторого фиксированного критического размера [4], что не изменит вид рассматриваемых ниже асимптотических решений.

Для не зависящих от $s$ коэффициентов захвата $\left(\sigma_{s}=\sigma_{0}\right)$ уравнения (1) и (2) с учетом $\sum_{s=0}^{\infty} n_{s}=N_{0}=$ const приводят к точному выражению для скорости нуклеации

$$
\frac{d N}{d t}=\frac{N_{0}}{t_{1}} \exp \left[-\frac{t-t_{0}\left(1-e^{-t / t_{0}}\right)}{t_{1}}\right]\left(1-e^{-t / t_{0}}\right) .
$$

Письма в ЖТФ, 2016, том 42, вып. 22 


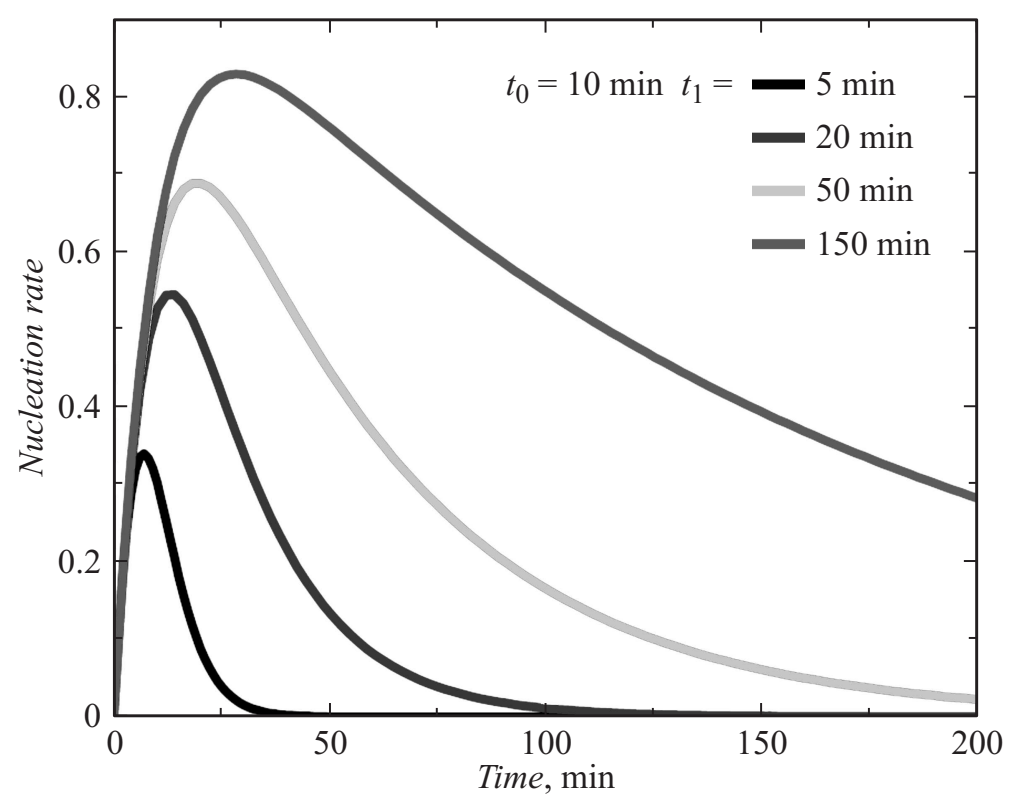

Безразмерная скорость нуклеации $\left(t_{1} / N_{0}\right) d N / d t$ в зависимости от общей продолжительности эпитаксиального процесса, полученная из выражения (3) с фиксированным $t_{0}=10 \mathrm{~min}$ и $t_{1}$, возрастающим от 5 до $150 \mathrm{~min}$.

Времена релаксации имеют вид

$$
t_{0}=\frac{t_{A}}{1+D \sigma_{0} N_{0} t_{A}}, \quad t_{1}=\frac{N_{0}}{I}\left(1+\frac{1}{D \sigma_{0} N_{0} t_{A}}\right) .
$$

Полученное решение содержит два характерных масштаба времени, влияющих на скорость нуклеации - время $t_{0}$, необходимое для начального заполнения поверхности галлием, и время $t_{1}$ фактической нуклеации кристаллического $\mathrm{GaN}$. Время $t_{0}$ не зависит от потока галлия и может лимитироваться либо десорбцией (при $\left.D \sigma_{0} N_{0} t_{A} \ll 1\right)$, либо захватом диффундирующих адатомов центрами нуклеации (при $\left.D \sigma_{0} N_{0} t_{A} \gg 1\right)$. Время $t_{1}$ всегда обратно пропорционально потоку галлия и лимитируется либо общим числом центров нуклеации (при $\left.D \sigma_{0} N_{0} t_{A} \ll 1\right)$, либо скоростью диффузии к ним (при $\left.D \sigma_{0} N_{0} t_{A} \gg 1\right)$. На рисунке показаны различные режимы нуклеации при фиксированном $t_{0}$

Письма в ЖТФ, 2016, том 42, вып. 22 
и возрастающем $t_{1}$, например, при уменьшении потока галлия. Наиболее представительный размер островков отвечает максимуму скорости нуклеации. Однако общее ВИ естественно определить как время, необходимое для нуклеации основной массы островков, при котором скорость нуклеации убывает на порядок, а плотность островков насыщается. Следовательно, в общем случае $t_{i n c}=t_{0}+t_{1}$. Зависимость ВИ от потока галлия $I$ имеет вид $c_{1}(T)+c_{2}(T) / I$, с некоторыми $c_{1}$ и $c_{2}$, являющимися функцией температуры.

Хотя десорбция галлия является весьма существенной при температурах $750-800^{\circ} \mathrm{C}$ [9], само по себе время $t_{A}$ не может составлять десятки или сотни минут и, следовательно, лимитировать ВИ. Более естественно считать, что плотность адатомов достигает максимального значения $I t_{A}$ за время порядка $t_{A}$ и затем спадает гораздо медленнее (со временем релаксации $t_{1} \gg t_{A}$ ) в связи с медленной нуклеацией островков $\mathrm{GaN}$. Такая ситуация характерна для режима неполной конденсации $[5,6]$ и в нашей модели соответствует условию $D \sigma_{0} N_{0} t_{A} \ll 1$ (высокая скорость десорбции, медленная поверхностная диффузия или малая концентрация центров нуклеации). В режиме неполной конденсации получаем $t_{0}=t_{A}$ и $t_{1}=t_{A} / v \gg t_{A}$. Параметр

$$
v=D \sigma_{0} I t_{A}^{2} \ll 1
$$

равен отношению характерной диффузионной площади $D \sigma_{0} t_{A}=\lambda^{2}$ (где $\lambda$ есть эффективная диффузионная длина адатомов галлия) к площади поверхности $\left(I_{A}\right)^{-1}$, приходящейся на один адатом при максимальной концентрации $I t_{A}$. В режиме неполной конденсации данный параметр много меньше единицы, и нуклеация лимитируется малой вероятностью встречи адатомов галлия из-за их интенсивной десорбции.

Случай $\sigma_{s}=\sigma_{0}$ отвечает диффузионному режиму роста трехмерных островков $\mathrm{GaN}$ [21]. Покажем теперь, что обратная пропорциональность ВИ потоку сохраняется при любой степенной зависимости $\sigma_{s}=\sigma_{*} s^{\alpha}$ с индексом $\alpha<1$ [21]. Вводя усреднение по нормированной функции распределения по размерам $\left\langle s^{\alpha}\right\rangle=N_{0}^{-1} \sum_{s=0}^{\infty} s^{\alpha} n_{s}$, замечаем, что в пределе больших времен $\left(n_{0} \rightarrow 0, n_{A} \rightarrow 0\right)$ справедливо $\langle s\rangle \cong(I t) / N_{0}$, т. е. средний размер островков равен отношению числа осажденных атомов галлия к числу центров нуклеации. Далее, имеем $\sigma_{*} \sum_{s=0}^{\infty} s^{\alpha} n_{s}=\sigma_{*} N_{0}\left\langle s^{\alpha}\right\rangle \cong c \sigma_{*} N_{0}\langle s\rangle^{\alpha}$, где значение $c$ порядка единицы

Письма в ЖТФ, 2016, том 42, вып. 22 
зависит от вида распределения по размерам и в целом используемое приближение справедливо для достаточно узких распределений [21]. Используя данные выражения в (2), получаем асимптотическое стационарное решение для концентрации адатомов

$$
n_{A} \cong \frac{I t_{A}}{1+D t_{A} c \sigma_{*} N_{0}^{1-\alpha}(I t)^{\alpha}} .
$$

Подставляя (6) в (1) и интегрируя, получаем

$$
n_{0} \cong \exp \left[-\frac{\sigma_{0}}{\sigma_{*} c(1-\alpha)}\left(\frac{I t}{N_{0}}\right)^{1-\alpha}\right] .
$$

Видно, что оба выражения (6) и (7) содержат время только в комбинации It. Это же справедливо и для скорости нуклеации $d N / d t \propto n_{A} n_{0}$, следовательно, ВИ обратно пропорционально I. Можно показать, что зависимость $t_{i n c} \propto I^{-p}$ появляется в случае гомогенной нуклеации, что будет предметом отдельного сообщения.

С использованием аррениусовских температурных зависимостей для коэффициента диффузии и времени десорбции галлия с энергиями активации $E_{D}$ и $E_{A}$ и предэкспонентами $D_{0}$ и $t_{A}^{0}$ соответственно наш основной результат для ВИ принимает вид

$$
t_{i n c}=\frac{1}{D_{0} t_{A}^{0} \sigma_{0}(T)} \frac{1}{I} \exp \left[-\frac{\left(E_{A}-E_{D}\right)}{k_{B} T}\right] .
$$

Поскольку барьер десорбции $E_{A}$ всегда больше барьера диффузии $E_{D}[5]$, полученное выражение имеет тот же вид, что и приведенное в начале статьи, с энергией активации $E=E_{A}-E_{D}$ и $p=1$, что качественно соответствует работам $[7,9,10]$. Отметим, что чисто аррениусовская температурная зависимость ВИ может нарушаться при учете зависимости $\sigma_{0}(T)$. Как уже указывалось, отличие степенного индекса потока $p$ от единицы свидетельствует о гомогенном характере нуклеации в данных экспериментальных условиях и не связано с критическим размером. Проведенное рассмотрение позволило расшифровать физические параметры, ответственные за температурную и потоковую зависимость ВИ, и определять эти параметры из измеряемых данных по ВИ.

Данная работа выполнена при поддержке гранта Российского научного фонда № 14-22-00018.

Письма в ЖТФ, 2016, том 42, вып. 22 


\section{Список литературы}

[1] Kashchiev D., Verdoes D., Van Rosmalen G.M. // J. Cryst. Growth. 1991. V. 110. P. 373.

[2] Venables J.A. // Sur. Sci. 1994. V. 299-300. P. 798.

[3] Thompson C.V. // J. Mater. Res. 1999. V. 14. P. 3161.

[4] Venables J.A., Spiller G.D.T., Hanbucken M. // Rep. Prog. Phys. 1984. V. 47. P. 399.

[5] Kukushkin S.A., Osipov A.V. // Prog. Surf. Sci. 1996. V. 51. P. 1.

[6] Dubrovskii V.G. // Phys. Stat. Sol. (b). 1992. V. 171. P. 345.

[7] Consonni V., Trampert A., Geelhaar L. et al. // Appl. Phys. Lett. 2011. V. 99. P. 033102.

[8] Hestroffer K., Leclere C., Cantelli V. et al. // Appl. Phys. Lett. 2012. V. 100. P. 212107.

[9] Fernández-Garrido S., Zettler J.K., Geelhaar L. et al. // Nano Lett. 2015. V. 15. P. 1930.

[10] Sobanska M., Korona K.P., Zytkiewicz Z.R. et al. // J. Appl. Phys. 2015. V. 118. P. 184303.

[11] Wagner R.S., Ellis W.C. // Appl. Phys. Lett. 1964. V. 4. P. 89.

[12] Sanchez-Garcia M.A., Calleja E., Monroy E. et al. // J. Cryst. Growth. 1998. V. 183. P. 23.

[13] Galopin E., Largeau L., Patriarche G. et al. // Nanotechnology. 2001. V. 22. P. 245606.

[14] Dubrovskii V.G., Consonni V., Trampert A. et al. // Phys. Rev. B. 2012. V. 85. P. 165317.

[15] Consonni V., Dubrovskii V.G., Trampert A. et al. // Phys. Rev. B. 2012 . V. 85. P. 155313.

[16] Dubrovskii V.G., Consonni V., Geelhaar L. et al. // Appl. Phys. Lett. 2012. V. 100. P. 153101.

[17] Dubrovskii V.G., Sibirev N.V., Zhang X. et al. // Cryst. Growth Des. 2010. V. 10. P. 3949.

[18] Zhang X., Dubrovskii V.G., Sibirev N.V. et al. // Cryst. Growth Des. 2011. V. 11. P. 5441.

[19] Cirlin G.E., Dubrovskii V.G., Petrov V.N. et al. // Semicond. Sci. Technol. 1998. V. 13. P. 1262.

[20] Дубровский В.Г., Бердников Ю.С., Соколова Ж.В. // Письма в ЖТФ. 2015. T. 41. B. 5. C. 73.

[21] Dubrovskii V.G. // J. Chem. Phys. 2009. V. 131. P. 164514.

Письма в ЖТФ, 2016, том 42, вып. 22 